\section{Effects of radiopharmaceuticals on articular cartilage's mechanical properties}

\begin{abstract}
As radiation science and technology advances, nuclear medicine applications are increasing worldwide which necessitate the understanding of biological implications of such practices. Ionizing radiation has been shown to cause degraded matrix and reduced proteoglycan synthesis in cartilage, and the late consequences of which may include degenerative arthritis or arthropathy. Although degenerative effects of the ionizing radiation on cartilage tissue have been demonstrated, the effects on the mechanical properties of articular cartilage are largely unknown. The radiopharmaceuticals, technetium-99m and technetium-99m sestamibi, were utilized on bovine articular cartilage to investigate these effects. We used two different mechanical tests to determine the mechanical properties of articular cartilage. Dynamic and static mechanical tests were applied to calculate compressive modulus for articular cartilage. We observed clearly higher control modulus values than that of experimental groups which account for lesser stiffness in the exposed cartilage. In conclusion, compressive moduli of bovine articular cartilage were found to decrease after radiopharmaceutical exposure, after both instantaneous and equilibrium mechanical experiments.
\end{abstract}

Keywords: articular cartilage $\bullet$ compressive modulus $\bullet$ technetium-99m • technetium-99m sestamibi $\bullet$ ionizing radiation

\author{
N. Kuzu, E. Cicek ${ }^{\bowtie}$ \\ Department of Physics \\ Faculty of Science \& Art \\ Mehmet Akif Ersoy University \\ Burdur, 15030 Turkey \\ E-mail: ekrcicek@gmail.com
}

Received: 31 August 2017

Accepted: 20 February 2019

\section{Introduction}

The main function of the articular cartilage, a hyaline cartilage that covers the ends of articulating bones, is to minimize the mechanical stresses during joint loading to protect the bone from damage enabling frictionless motion among the joint surfaces and absorbing the load [1-3].

Ionizing radiation can cause a severe acute and persistent reduction in the structural integrity of irradiated skeletal tissue [4, 5]. Although largely masked by the considerable delay existing between radiation exposure and joint symptoms, these effects have also been shown to be responsible for progressive articular cartilage degeneration [6]. Considered to be late consequences of ionizing radiation exposure, degenerative arthritis or arthropathy observed in the knee is generally attributed to osteonecrosis-induced cartilage degradation [7].

Since it is an avascular tissue with low intracellular oxygen levels [1], the cartilage tissue has inherent cell radiation sensitivity and poor impairment capabilities. The radiation response of articular cartilage is hence characterized by an active degradation of cartilage matrix and reduced proteoglycan production $[8,9]$. However, the exact mechanisms through which the articular tissues are affected by ionizing radiation have not been identified. 
Only a few studies on effects of ionizing radiation on articular cartilage mechanical properties are presented [7]. It was shown that irradiation can cause an acute reduction in the surface mechanical properties of articular cartilage, specifically lowering compressive stiffness $[7,10]$.

The principal components of articular cartilage are collagen, proteoglycan, and water [11]. Mechanical properties of cartilage are determined by cartilage matrix that consists mainly of type II collagen and proteoglycans [12]. Compressive modulus reduction can be seen in irradiated cartilage when radiation changes the metabolism of cartilage matrix. Active degradation of proteoglycans or lowered proteoglycan or collagen II synthesis [7] and diminishing proteoglycan content in cartilage matrix [9] can lower tissue's compressive stiffness. In addition, collagen II synthesis has been shown to be lowered in articular chondrocytes following radiation exposure in bovine articular cartilage [13].

Preventing radiation-induced complications has led to a renewed interest in the understanding of the specific effects of radiation exposure on cartilage [1]. Although various reports demonstrate cartilage damage following X-ray or gamma-ray exposure, radiopharmaceutical effects on articular cartilage are relatively unknown. Radiopharmaceuticals in nuclear medicine are increasingly used to diagnose and treat diseases such as cancer and cardiovascular diseases. The goal of the present study was to investigate the effects of radiopharmaceuticals on the mechanical properties of bovine articular cartilage. Dynamic (instantaneous) and static (equilibrium) mechanical tests were applied to calculate compressive modulus for articular cartilage.

\section{Materials and methods}

Articular cartilage samples that were taken from fresh mature bovine tibiae were obtained from a local abattoir in the same day after slaughter (Burdur, Turkey). After removing the surrounding soft tissue, osteochondral explants $(\sim 5 \mathrm{~mm} \times 5 \mathrm{~mm}$ width) were harvested from the lateral tibial plateau and transported in phosphate-buffered saline (PBS) solution. Articular cartilage samples were taken from specimens that displayed no macroscopic degeneration.

Cartilage samples were placed inside $3.33 \mathrm{GBq}$ technetium-99m and $2.035 \mathrm{GBq}$ technetium-99m sestamibi samples for a week, while the control samples were soaked in physiological saline solution for the same period in the experimental group. All samples were stored at refrigerator temperature. After a week, both sample groups were taken for compression tests.

Two different mechanical tests, instantaneous and equilibrium, were applied to calculate compressive moduli.

The information about the alignment of collagen fibers was obtained, and the dimensions required for calculating compressive stresses were determined from polarized light microscopy images using a Nikon Eclipse E200 PLM.
The dimensions required for calculating compressive stresses were determined from light microscopy (Olympus CX41) images.

\section{Compression tests for instantaneous modulus}

For comparison, the LR5K Plus universal tensile testing machine and a $3 \mathrm{~cm}$ diameter homemade cylindrical compression holder were used. Unconfined compression tests were performed on cartilage samples up to $70 \%$ strain.

A total of 56 samples were subjected to compression: 16 control group samples, 20 technetium-99m-exposed samples, and 20 technetium-99m sestamibi-exposed samples.

The samples were placed in the designed tester device, and the resulting diagrams were obtained for the LR5K Plus tester [14]. The test results were drawn as diagrams in NEXYGEN Plus Data Analysis Software and MS Excel 2010. The vertical axis given in the diagrams is the stress values (MPa), while the horizontal axis shows the strain values.

\section{Compression tests for equilibrium modulus}

A stress relaxation test was performed using a cylindrical, plane-ended 3-mm-diameter indenter positioned perpendicular to the superficial zone. Force and displacement were logged using Picolog (Pico Technology Ltd.). A 50-kg load cell (NMNC50L, CAS Corporation, South Korea) was used to apply force $(1 \mathrm{mV}=46.166 \mathrm{~N})$. Initially, a force was applied and the cartilage was allowed to come to the equilibrium. The cartilage surface was displaced $0.7 \mathrm{~mm}$ in total in ten steps, each step being $70 \mu \mathrm{m}$.

Stress was calculated as load per unit macroscopic sectional field. Strain was calculated with reference to the relaxed length of the sample. Modulus was determined from the linear slope of the stress-strain curve.

The control and irradiated samples were immersed in a saline-filled water bath, throughout testing in room temperature. Eight samples, six irradiated and two identical control samples, were analysed in total. Six control samples, seven samples exposed to 3.33-GBq technetium-99m, and nine samples exposed to 2.035-GBq technetium-99m sestamibi were analysed. The details related to the compression method can be found in the previous study [15].

One-way analysis of variance (ANOVA) was used for statistical analysis. A $p$-value of $<0.05$ was considered significant.

\section{Results and discussion}

Both instantaneous experiments and equilibrium experiments, the moduli values of the control groups showed clear differences with that of the technetium$-99 \mathrm{~m}$ and sestamibi exposed experimental groups.

In instantaneous experiments, the highest and lowest stress values are 6.5 $\mathrm{MPa}$ and $3.6 \mathrm{MPa}$, re- 


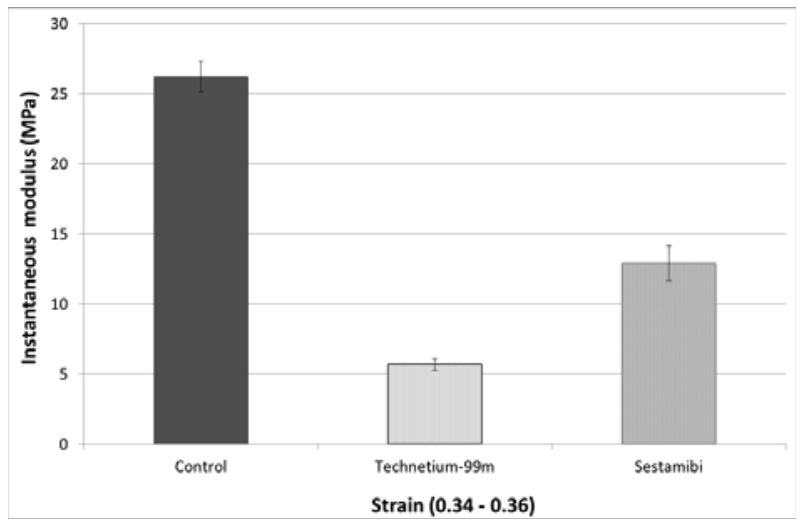

Fig. 1. Instantaneous modulus graph of the control and the experimental (technetium-99m and sestamibi) groups.

spectively, for 0.35 strain for control. The highest and lowest stress values are $2.4 \mathrm{MPa}$ and $0.8 \mathrm{MPa}$, respectively, for 0.35 strain for technetium-99m. The average modulus of the control samples was statistically higher than the average modulus of technetium-99m. It means that the technetium-99m group has less stiffness than the control group. This result indicates that technetium-99m induced the decline in cartilage.

For 0.35 strain, the highest stress value is $5.9 \mathrm{MPa}$, while the lowest stress value is $0.8 \mathrm{MPa}$ for control and sestamibi (MIBI, methoxyisobutylisonitrile). Likewise, control-technetium-99m that slopes of control curves higher than slopes of MIBI curves. So, articular cartilage has less stiffness after exposed to MIBI.

Instantaneous compressive modulus values for the strain $(0.34-0.36)$ were obtained from the stress-strain curves. These values are $26.21 \pm 1.09$ for control, $5.69 \pm 0.40$ for technetium-99m, and $12.91 \pm 1.25$ for sestamibi. The modulus values were clearly higher in the control group as compared to the experimental (technetium-99m and sestamibi) group (Fig. 1). These differences were significant ( $p$ is almost 0 ).

In equilibrium experiments, the relaxation time in the control group is longer than the experimental group. Equilibrium compressive modulus values for the strain $(0.24-0.32)$ were obtained from the stress-strain curves. These are $29.13 \pm 9.78,26.60 \pm$ 7.24 , and $9.89 \pm 2.54$ for control, technetium-99m,

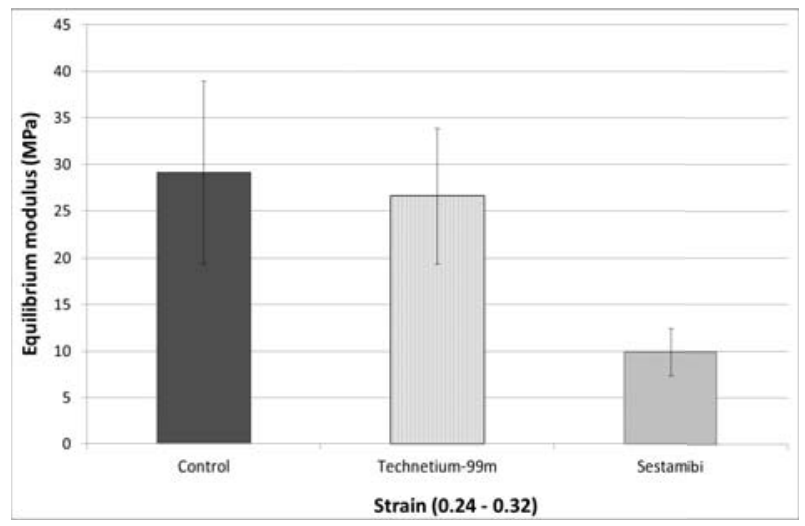

Fig. 2. Equilibrium modulus graph of the control and the experimental (technetium-99m and sestamibi) groups. and sestamibi, respectively. The modulus values were higher in the control group as compared to the experimental (technetium-99m and sestamibi) group (Fig. 2). These differences were insignificant $(p=0.077)$.

The modulus values of the control group were higher than those of the radiopharmaceutical-exposed experimental group for both instantaneous and equilibrium experiments. The meaning of these results is that the strength of the articular cartilage is negatively affected by the radiopharmaceuticals for both experiments.

\section{Conclusions}

Compressive modulus of bovine articular cartilage was found to decrease after radiopharmaceutical exposure to ionizing radiation, both instantaneous and equilibrium mechanical experiments. We document that radiopharmaceuticals cause reduction in the stiffness of the bovine articular cartilage.

Acknowledgment. This research has been supported by Mehmet Akif Ersoy University, Scientific Research Fund (project no. 0359-YL-16).

\section{References}

1. Saintigny, Y., Cruet-Hennequart, S., Hamdi, D. H., Chevalier, F., \& Lefaix, J. L. (2005). Impact of therapeutic irradiation on healthy articular cartilage. Radiat. Res., 183(2), 135-146. DOI: 10.1667/ RR13928.1.

2. Padalkar, M. V., Spencer, R. G., \& Pleshko, N. (2013). Near infrared spectroscopic evaluation of water in hyaline cartilage. Ann. Biomed. Eng., 41(11), 2426-2436. DOI: 10.1007/s10439-013-0844-0.

3. Li, G., Thomson, M., Dicarlo, E., Yang, X., Nestor, B., Bostrom, M. P. G., \& Camacho, N. P. (2005). A chemometric analysis for evaluation of early-stage cartilage degradation by infrared fiber-optic probe spectroscopy. Appl. Spectrosc., 59(12), 1527-1533. DOI: $10.1366 / 000370205775142593$.

4. Baxter, N. N., Habermann, E. B., Tepper, J. E., Durham, S. B., \& Virnig, B. A. (2005). Risk of pelvic fractures in older women following pelvic irradiation. JAMA, 294(20), 2587-2593. DOI: 10.1001/jama.294.20.2587.

5. Willey, J. S., Livingston, E. W., Robbins, M. E., Bourland, J. D., Tirado-Lee, L., Smith-Sielicki, H., \& Bateman, T. A. (2010). Risedronate prevents early radiation-induced osteoporosis in mice at multiple skeletal locations. Bone, 46(1), 101-111. DOI: 10.1016/j.bone.2009.09.002.

6. Kolár, J., Vrabec, R., \& Chyba, J. (1967). Arthropathies after irradiation. J. Bone Joint Surg. Am., 49(6), 1157-1166. DOI: 10.2106/00004623-19674906000013.

7. Lindburg, C. A., Willey, J. S., \& Dean, D. (2013) Effects of low dose X-ray irradiation on porcine articular cartilage explants. J. Orthop. Res., 31(11), 1780-1785. DOI: $10.1002 /$ jor.22406. 
8. Hugenberg, S. T., Myers, S. L., \& Brandt, K. D. (1989). Suppression of glycosaminoglycan synthesis by articular cartilage, but not of hyaluronic acid synthesis by synovium, after exposure to radiation. Arthritis. Rheum., 32(4), 468-474. DOI: 10.1002/ anr.1780320417.

9. Willey, J. S., Long, D. L., Vanderman, K. S., \& Loeser, R. F. (2013). Ionizing radiation causes active degradation and reduces matrix synthesis in articular cartilage. Int. J. Radiat. Biol., 89(4), 268-277. DOI: 10.3109/09553002.2013.747015.

10. Lindburg, A. B., Willey, J. S., DesJardins, D. J., \& Dean, D. (2011). Effect of X-ray irradiation on porcine and murine cartilage modulus. In Society for Biomaterials 2011 Annual Meeting and Exposition (abstract \#794). Available from http://abstracts.biomaterials. org/data/papers/2011/794.pdf.

11. Yin, J., Xia, Y., \& Lu, M. (2012). Concentration profiles of collagen and proteoglycan in articular cartilage by Fourier transform infrared imaging and principal component regression. Spectroc. Acta Pt A-Mol. Biomolec. Spectr., 88, 90-96. DOI: 10.1016/j. saa.2011.12.002.
12. Long, D. L., \& Loeser, R. F. (2010). P38gamma mitogen-activated protein kinase suppresses chondrocyte production of MMP-13 in response to catabolic stimulation. Osteoarthr. Cartil., 18(9), 1203-1210. DOI: 10.1016/j.joca.2010.05.016.

13. Ailland, J., Kampen, W. U., Schünke, M., Trentmann, J., \& Kurz, B. (2003). Beta irradiation decreases collagen type II synthesis and increases nitric oxide production and cell death in articular chondrocytes. Ann. Rheum. Dis., 62(11), 1054-1060. DOI: 10.1136/ ard.62.11.1054.

14. Cicek, E., \& Cakmak, E. (2018). Hydrogen peroxide induced oxidative damage on mineral density and mechanical properties of bone. Brazilian Archives of Biology and Technology, 61, e18180043. DOI: 10.1590/1678-4324-2018180043.

15. Cicek, E. (2016). Effect of X-ray irradiation on articular cartilage mechanical properties. Acta Phys. Pol. A, 129(2), 200-202. DOI: 10.12693/APhysPo1A.129.200. 